\title{
Superior Mesenteric Artery Embolus as a Delayed Complication of a Cocaine Induced Myocardial Infarction
}

\author{
Kearsley $\mathrm{R}^{{ }^{1}}$, Osman $\mathrm{AME}^{2}$, Barnwell $\mathrm{N}^{1}$, Medani $\mathrm{M}^{3}$ and Kavanagh $\mathrm{E}^{4}$ \\ ${ }^{1}$ Specialist Registrar in Anaesthesia and Intensive Care Medicine, University Hospital Limerick, Ireland \\ ${ }^{2}$ Senior House Officer in Vascular Surgery, University Hospital Limerick, Ireland \\ ${ }^{3}$ Specialist Registrar in Vascular Surgery, University Hospital Limerick, Ireland \\ ${ }^{4}$ Consultant Vascular Surgeon, University Hospital Limerick, Ireland
}

*Corresponding author: Kearsley R, Specialist Registrar in Anaesthesia and Intensive Care Medicine, University Hospital Limerick, Ireland, E-mail: rosemariekearsley@gmail.com

Citation: Kearsley R, Osman AME, Barnwell N, Medani M, Kavanagh E (2018) Superior Mesenteric Artery Embolus as a Delayed Complication of a Cocaine Induced Myocardial Infarction. J Case Rep Stud 6(2): 201. doi: 10.15744/2348-9820.6.201

Received Date: February 06, 2018 Accepted Date: April 25, 2018 Published Date: April 27, 2018

\begin{abstract}
Cocaine has acute and chronic effects on health as well as addiction and dependence and places a significant burden on healthcare systems. After cannabis, it is the most frequently used illicit drug worldwide. It is estimated that between 14 and 21 million people use the drug each year [1]. Its main adverse effects occur in the cardiovascular system. Cocaine causes sympathomimetic actions such as tachycardia, hypertension and coronary artery constriction due to enhancement of release of catecholamines. It enhances thrombus formation by activating platelets and potentiating thromboxane production [2]. The consequences of these effects include chest pain, myocardial infarction, aortic dissection, coronary artery aneurysm, myocarditis, cardiomyopathies and arrhythmias. Our case demonstrates a young patient who had a delayed presentation of a myocardial infarction, in the setting of recent cocaine use that was complicated by an apical thrombus which resulted in distal emboli to his superior mesenteric artery and left renal artery. It highlights the fact that in a young patient with no other risk factors how complications may arise, even weeks after cocaine use.

Keywords: Cocaine; Thrombus; Myocardial infarct; Embolectomy; Echocardiography
\end{abstract}

\section{Introduction}

We present the case of a young man who presented to the emergency department with severe abdominal pain of 5 days duration. Computed tomography (CT) of his abdomen demonstrated a superior mesenteric artery (SMA) occlusion with bowel ischaemia as well as a moderate renal infarct. Investigations for clotting disorders were all negative and no haematological cause was found for his presentation. The patient had admitted to use of cocaine approximately ten days prior to admission with a palpitationlike episode after use. Transthoracic echocardiography performed on admission, demonstrated a left ventricular thrombus. Two weeks following his initial presentation a cardiac magnetic resonance imaging (MRI) study demonstrated a transmural infarction from base to apex without evidence of any residual thrombus. The timing of thrombus development and findings on cardiac MRI correlated with his use of cocaine prior to presentation to hospital.

\section{Case Report}

A 25-year-old male, with a past medical history of cocaine and cannabis use, presented to the emergency department with a 5-day history of abdominal pain, diarrhoea and vomiting. His abdominal pain was generalised, not localising anywhere and radiated to his back. His family history was unremarkable for cardiac disease. Regarding his social history, he smoked one packet of cigarettes per week, drank alcohol socially and used cocaine and cannabis on a weekly basis. He last used cocaine 10 days prior to admission.

On admission his vital signs were a blood pressure of $111 / 72 \mathrm{mmHg}$, heart rate of 112 beats per minute, respiratory rate of 24 breaths per minute, temperature of $37.1{ }^{\circ} \mathrm{C}$ and his oxygen saturations were $98 \%$ on room air. On examination he was pale and diaphoretic with a rigid abdomen.

Laboratory investigations demonstrated haemoglobin of $15.4 \mathrm{~g} / \mathrm{dL}$, a white cell count of 7.5, platelets of 354 . His biochemistry profile demonstrated sodium of 123 , a potassium of 3.1, a urea of 6.9, a creatinine of 78, a glucose of 6.7. His C- reactive protein was elevated at 173 . His initial venous blood gas showed a $\mathrm{pH}$ of 7.53 , a pCO2 of $3.96 \mathrm{kPa}$ and a lactate of $1.4 \mathrm{mmol} / \mathrm{L}$. 
He had an electrocardiogram (ECG) that showed a sinus tachycardia and QRS abnormalities consistent with an anteroseptal infarct. A chest x-ray was performed which was unremarkable and did not demonstrate a pneumoperitoneum. An urgent computed tomography (CT) of the abdomen and pelvis demonstrated an oedematous small bowel consistent with diffuse enteritis, a large filling defect in the superior mesenteric artery (SMA) consistent with a large embolism and a moderate sized renal infarct It also showed a left ventricular thrombus (Figure 1).

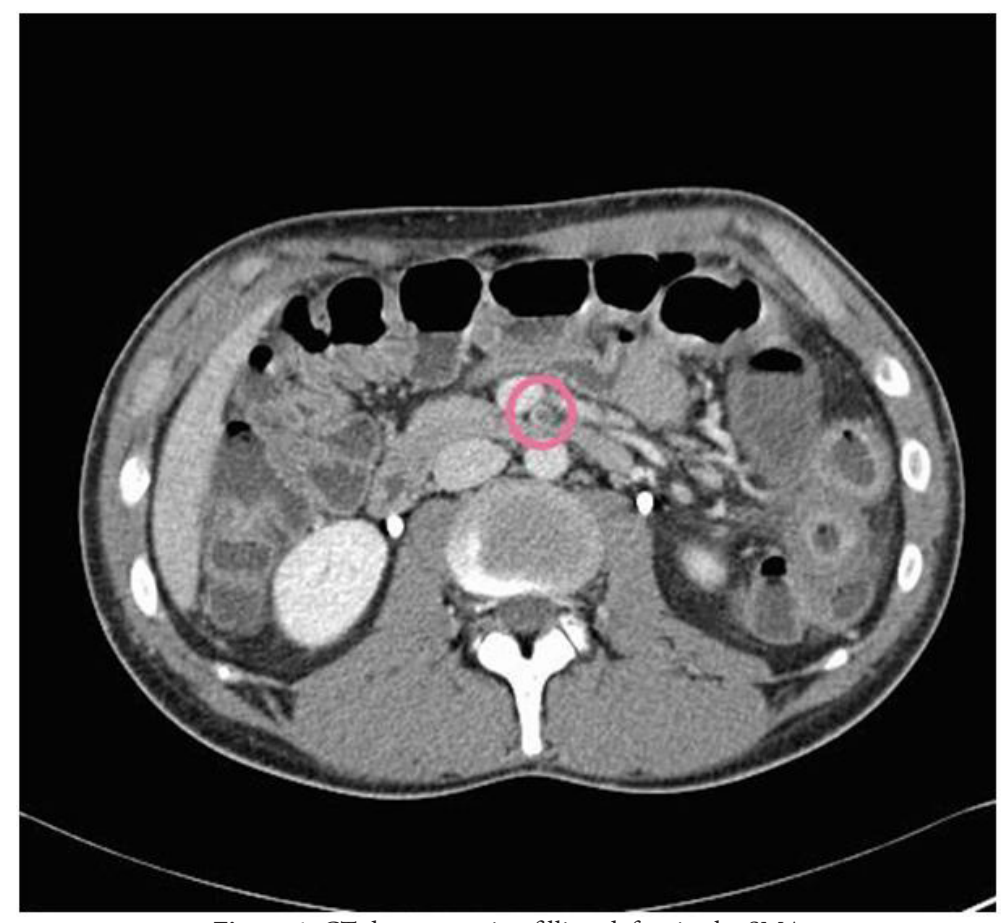

Figure 1: CT demonstrating filling defect in the SMA

He underwent an urgent laparotomy, which demonstrated 3 areas of patchy transmural necrosis and an occluded SMA with no Doppler signal. An SMA embolectomy was performed and flow was restored. The areas of necrosis were oversewn. He was transferred to the ICU and commenced on intravenous heparin to maintain an Activated Partial Thromboplastin Time (APTT) of between 60 and 80 .

In ICU, he had a TTE was performed to further evaluate the left ventricular thrombus which had been seen on the initial CT to assess for a source of the distal emoboli to the SMA and the renal artery. This TTE demonstrated moderate anterior wall hypokinesis and a large laminar thrombus along the anterior wall (Figure 2). He had cardiac enzymes measured that showed an elevated troponin of greater than 1000, consistent with myocardial injury. He remained in ICU for monitoring for 4 days on intravenous heparin before being transferred to the high dependency unit, where he spent a further 4 days. On day 8 he was discharged to the ward on therapeutic tinzaparin at a dose of 175 anti-Xa IU/kg daily. On day 14 of admission, he underwent a magnetic resonance imaging of his heart with contrast. This demonstrated a transmural infarction at anterior, anteroseptal and anterolateral walls from base to apex without evidence of an apical thrombus. The territory of the left anterior descending artery was found to be non-viable.

He was discharged home from the hospital on rivaroxaban and aspirin. He was reviewed at both the surgical and cardiac outpatient clinics and had no further complications.

\section{Discussion}

In this case, we see an otherwise healthy 25 year old man present with complications of distal emboli from a left ventricular thrombus. Prior to admission he used cocaine in the preceding fortnight, and admitted to some palpitation-like episodes after use. We suspect, based on his echocardiogram and MRI findings, that he had a cocaine-related cardiac event, which due to his delayed presentation and therefore lack of anti-coagulation led to the formation of a left ventricular thrombus. Myocardial ischaemia is a well-known complication of cocaine use [3], as is cocaine induced mesenteric ischaemia. However, mesenteric ischaemia caused by a ventricular thrombus due to infarcted hypokinetic cardiac muscle from cocaine has not previously been described. A case of development of intra-cardiac thrombus post cocaine induced myocardial ischaemia with no subsequent complications has been reported however [4]. Acute mesenteric ischaemia is a serious and potentially fatal vascular emergency. Prompt recognition, diagnosis and intervention are required to prevent bowel necrosis and patient death. Overall mortality of acute mesenteric ischaemia is $60-80 \%$. Arterial thromboembolic phenomena account for the majority of cases of acute mesenteric ischaemia. Typical patients are over 60 years and have a history of an arrhythmia or a previous myocardial infarction. Presentation 
in a young patient with no antecedent cardiac symptoms is unusual [5]. Given current widespread cocaine use, and the potentially fatal outcome associated with delayed diagnosis, intra-arterial thrombus causing mesenteric ischaemia should be considered in a patient with a history of cocaine use.

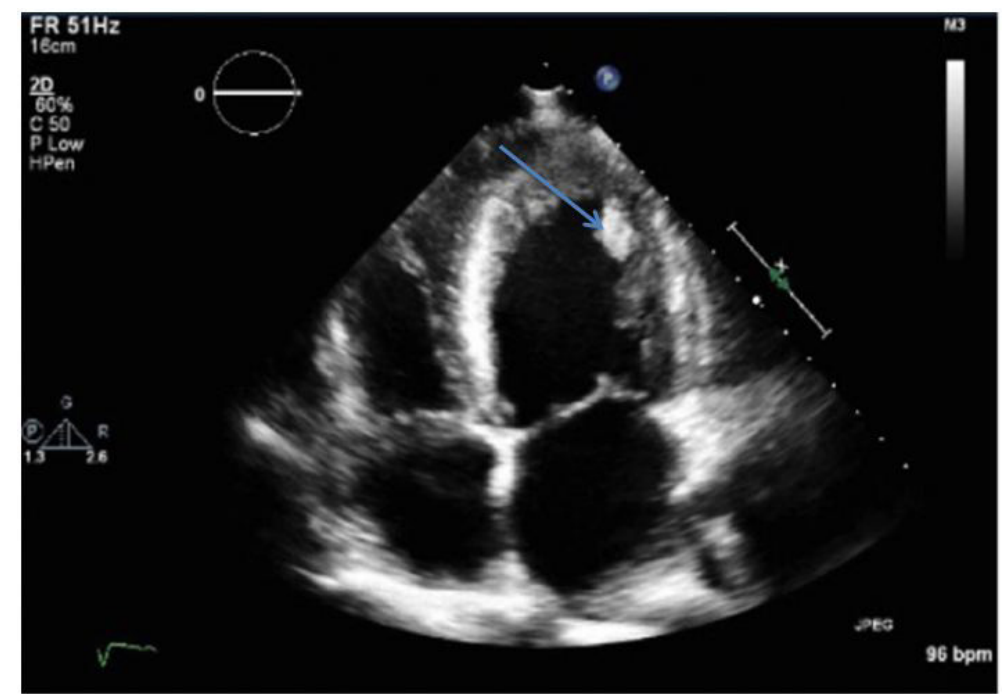

Figure 2: Transthoracic echocardiogram demonstrating a large thrombus in the left ventricle

\section{References}

1. Pomara C, Cassano T, D'Errico S, Bello S, Romano AD, et al. (2012) Data available on the extent of cocaine use and dependence: biochemistry, pharmacologic effects and global burden of disease of cocaine abusers. Curr Med Chem 19: 5647-57.

2. McCord J, Jneid H, Hollander JE, de Lemos JA, Cercek B, et al. (2008) Management of Cocaine-Associated Chest Pain and Myocardial Infarction. a scientific statement from the American Heart Association Acute Cardiac Care Committee of the Council on Clinical Cardiology. Circulation 117: 1897-907.

3. Maraj S, Figueredo VM, Lynn Morris D (2010) Cocaine and the heart. Clin Cardiol 33: 264-9.

4. Khan R, Archeda S, Jehangira W, Sen S, Yousif A (2016) Cocaine-Induced Delayed Myocardial Infarction Complicated by Apical Thrombus. J Clin Med Res 8: 59-61.

5. Oldenburg WA, Lau LL, Rodenberg TJ, Edmonds HJ, Burger CD (2004) Acute Mesenteric Ischaemia, A Clinical Review. Arch Intern Med 164: $1054-62$.

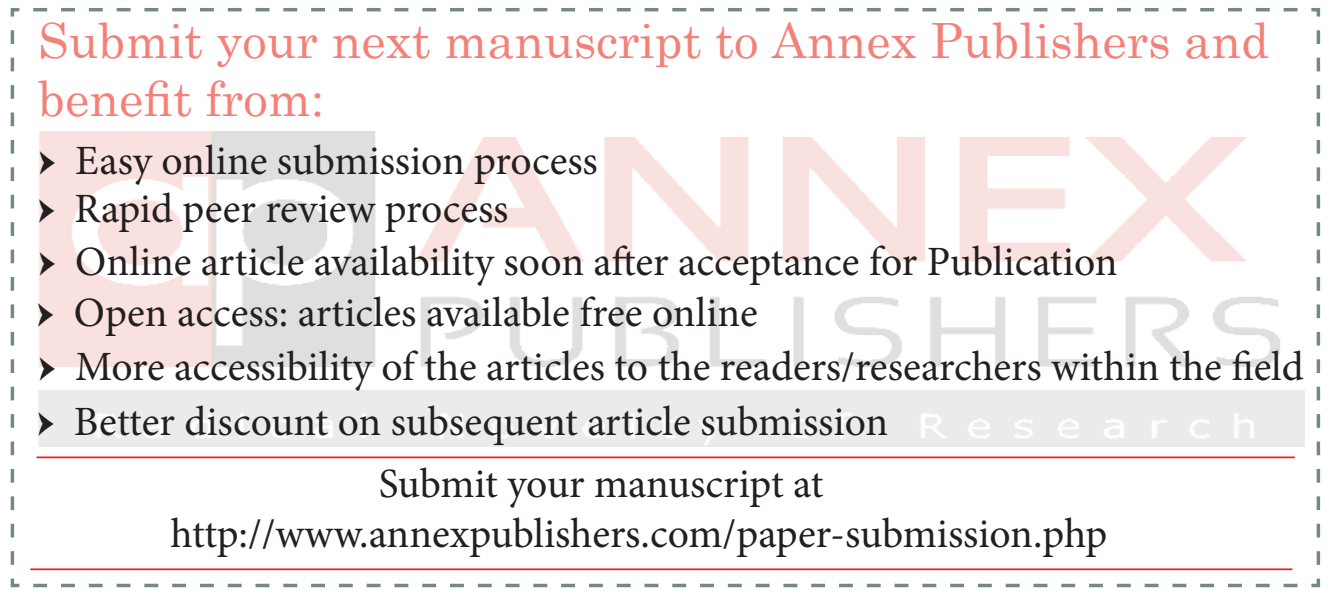

